\title{
Myelodysplastic syndrome with genetic predisposition
}

\author{
Meerim Park \\ Department of Pediatrics, Center for Pediatric Cancer, National Cancer Center, Goyang, Korea
}

p-ISSN 2287-979X / e-ISSN 2288-0011

https://doi.org/10.5045/br.2021.2020327

Blood Res 2021;56:S34-S38.

Received on December 21, 2020

Accepted on February 3, 2021

\section{Correspondence to}

Meerim Park, M.D, Ph.D.

Department of Pediatrics, Center for

Pediatric Cancer, National Cancer Center,

323 Ilsan-ro, Ilsandong-gu, Goyang 10408,

Korea

E-mail: meerim@ncc.re.kr

(C) 2021 Korean Society of Hematology

\begin{abstract}
Myelodysplastic syndrome (MDS) refers to a heterogeneous group of clonal blood disorders characterized by ineffective hematopoiesis, cytopenia, dysplasia, and an increased risk of acute myeloid leukemia (AML). A growing number of inherited genetic loci that contribute to MDS/AML development are rapidly being identified. As genetic sequencing has become increasingly integrated into clinical practice, clearly defined syndromes have emerged, known as the MDS/AML predisposition syndrome. With more patients and families being identified with predisposing conditions, knowledge of the approach of evaluating and managing MDS with genetic predisposition is increasingly essential. This article reviews MDS with genetic predisposition and the practical aspects of management in patients with predisposition syndrome.
\end{abstract}

Key Words Myelodysplastic syndrome, Genetic predisposition

\section{INTRODUCTION}

Myelodysplastic syndrome (MDS) is a collection of clonal hematopoietic disorders characterized by peripheral cytopenia, ineffective hematopoiesis, and an increased risk of progression to acute myeloid leukemia (AML) [1]. It is an increasingly prevalent disease, predominantly affecting patients of advanced age. The median age of onset is the $7^{\text {th }}$ decade, while it is rare in children and young adults. Childhood MDS has been historically categorized as de novo or primary MDS and secondary MDS, defined as MDS from inherited bone marrow failure (BMF) syndromes, acquired BMF, and therapy-related MDS associated with previous cytotoxic therapy [2]. Based on recent advances in the defining mechanisms of leukemogenesis and the role of clonal hematopoiesis as a predisposing factor for myeloid malignancies, there is increasing evidence indicating a potential underlying predisposition syndrome in MDS that was previously considered de novo or primary. Recently, there has been a growing awareness of non-syndromic familial MDS predisposition syndromes, including those caused by gene mutations such as ANKRD6, GATA2, ETV6, RUNX1, etc. [3]. Recognizing patients with potential hereditary syndromes could provide valuable insights for genetic counseling and disease treatment. The increasing awareness of these conditions coupled with efforts to refer for genetic counseling and genetic testing has revealed that as many as $10 \%$ of individuals with hematologic malignancies may carry a germline susceptibility [4]. In recognition of the impact of this growing field on clinical care, the World Health Organization, European Leukemia Net, and National Comprehensive Cancer Network have all recently incorporated the consideration of MDS/ AML germline predisposition syndromes into the MDS/AML classification and clinical management guidelines, making knowledge of these syndromes now essential for clinicians [5-7].

\section{MDS with genetic predisposition (Table 1)}

Familial platelet disorder with predisposition to myeloid malignancy: Familial platelet disorder with predisposition to myeloid malignancy (FPDMM) is an autosomal dominant familial MDS/AML syndrome caused by inherited mutations in the hematopoietic transcription factor RUNX1. It typically presents with mild to moderate thrombocytopenia with normal-sized platelets, functional platelet defects leading to prolonged bleeding, and an increased risk of development to MDS, AML, or T-cell acute lymphoblastic leukemia. Recent data suggest that clonal hematopoiesis can be detected in $>80 \%$ of asymptomatic FPDMM individuals by age 50 , providing future means of disease surveillance [8].

The MDS/AML transformation rate in FPDMM is estimated to be $20-60 \%$, with a high degree of variability within families [9]. Although not definitely established, progression 
Table 1. Myelodysplastic syndrome with genetic predisposition.

\begin{tabular}{|c|c|c|c|c|c|c|}
\hline Syndrome & Pathogenesis & Inheritance & Known genes & Non-hematologic findings & Screening test & $\begin{array}{c}\text { Risk of } \\
\text { MDS/AML }\end{array}$ \\
\hline $\begin{array}{l}\text { Familial platelet } \\
\text { disorder with } \\
\text { predisposition to } \\
\text { myeloid malignancy }\end{array}$ & $\begin{array}{l}\text { Transcription } \\
\text { regulation }\end{array}$ & $\mathrm{AD}$ & RUNX1 & None & RUNX1 sequencing & $35 \%$ \\
\hline $\begin{array}{l}\text { ANKRD26-related } \\
\text { thrombocytopenia }\end{array}$ & $\begin{array}{l}\text { MAPK } \\
\text { signaling }\end{array}$ & $\mathrm{AD}$ & ANKRD26 & None & ANKRD26 sequencing & $8 \%$ \\
\hline $\begin{array}{l}\text { GATA2 } \\
\text { haploinsufficiency }\end{array}$ & $\begin{array}{l}\text { Transcription } \\
\text { regulation }\end{array}$ & $\mathrm{AD}$ & GATA2 & $\begin{array}{l}\text { MonoMac syndrome } \\
\text { (Monocytopenia, non-tuberculous } \\
\text { mycobacterial and viral infections), } \\
\text { Emgerger syndrome (lymphedema } \\
\text { and monosomy 7), cutaneous } \\
\text { warts, deafness }\end{array}$ & $\begin{array}{l}\text { GATA2 sequencing; } \\
\text { bone marrow } \\
\text { morphology and flow } \\
\text { cytometry }\end{array}$ & $50 \%$ \\
\hline $\begin{array}{l}\text { DDX41- associated } \\
\text { familial MDS/AML } \\
\text { syndrome }\end{array}$ & $\begin{array}{l}\text { DEAD/H-box } \\
\text { helicase }\end{array}$ & $\mathrm{AD}$ & DDX41 & $\begin{array}{l}\text { Long latency; presentation in }>40 \\
\text { years old adults with high risk MDS } \\
\text { and AML }\end{array}$ & DDX41 sequencing & Unknown \\
\hline $\begin{array}{l}\text { SRP72-associated } \\
\text { MDS }\end{array}$ & $\begin{array}{l}\text { SRP72 } \\
\text { transcription } \\
\text { factor }\end{array}$ & $\mathrm{AD}$ & SRP72 & Congenital nerve deafness & SRP72 sequencing & Unknown \\
\hline $\begin{array}{l}\text { ETV6-associated } \\
\text { familial } \\
\text { thrombocytopenia } \\
\text { and hematologic } \\
\text { malignancy }\end{array}$ & $\begin{array}{l}\text { ETV6 } \\
\text { transcription } \\
\text { factor }\end{array}$ & $\mathrm{AD}$ & ETV6 & $\begin{array}{l}\text { Thrombocytopenia, bleeding, } \\
\text { macrocytosis, possible association } \\
\text { with myopathy, GERD, esophageal } \\
\text { stricture, reading disability }\end{array}$ & ETV6 sequencing & Unknown \\
\hline
\end{tabular}

to MDS/AML can be associated with the acquisition of a second mutation in RUNX1 or an acquisition of trisomy 21 , consistent with the need for a "second-hit" event to initiate malignant transformation $[10,11]$.

ANKRD26-related thrombocytopenia: ANKRD26-related thrombocytopenia (ANKRD26-RT) is characterized by lifelong mild-to-moderate thrombocytopenia with a normal platelet size and no syndromic associations. Most individuals have normal hemostasis or a mild bleeding phenotype and do not present with severe spontaneous bleeding. The risk for myeloid malignancies, including MDS, AML, and chronic myelogenous leukemia (CML), is increased in individuals with ANKRD26 pathogenic variants [12]

Bone marrow morphology in affected individuals may demonstrate dysmegakaryopoiesis with hypolobulated micromegakaryocytes at baseline, presenting a diagnostic challenge of appropriately distinguishing individuals with germline ANKRD27 mutations versus dysmegakaryopoiesis related to the development of MDS [13]. Among the roughly 222 reported cases of ANKRD26-RT to date, there has been an increased incidence of myeloid malignancies, with $4.9 \%$ of patients developing acute leukemias, 2.2\% developing MDS, and 1.3\% developing CML, yielding an estimated 23-fold, 12-fold, and 21-fold risk for these malignancies, respectively, than the general population [14].

Familial MDS with mutated GATA2 (GATA2 deficiency): Germline mutation in GATA2 can lead to GATA2 deficiency, characterized by a complex multi-system disorder with various manifestations, including variable cytopenias, bone mar- row failure, MDS/AML, and severe immunodeficiency. To date, around 550 cases with germline GATA2 mutations have been reported in the literature. GATA2 deficiency is a highly penetrant disorder with a progressive course that often rapidly necessitates hematopoietic stem cell transplantation (HSCT) [15].

Describing the presenting symptoms of 57 patients with germline GATA2 deficiency from the National Institutes of Health (NIH) cohort, viral infections were seen in $23 \%$, disseminated non-tuberculosis mycobacterial infections in $28 \%$, MDS/AML in $21 \%$, lymphedema in $9 \%$, and invasive fungal infections in $4 \%$ [16]. According to a recent analysis of 25 patients with germline GATA2 mutation, MDS-associated mutations were identified in symptomatic GATA2 patients, including those presenting with overt MDS and with hypocellular/aplastic bone marrows without definitive dysplasia [17]. Healthy relatives of probands harboring the same germline GATA2 mutations had essentially normal marrows devoid of MDS-associated mutations. The findings suggest that abnormal clonal hematopoiesis is a common event in symptomatic germline-mutated GATA2 patients with MDS and those with hypocellular marrows without overt morphologic evidence of dysplasia, possibly indicating a pre-MDS stage warranting close monitoring for disease progression.

The management of patients with GATA2 deficiency often involves a multidisciplinary care team due to the multi-organ involvement. The high incidence of MDS/AML in these individuals warrants regular evaluation of the blood to check 
for signs of worsening immunodeficiency and monitor the blood counts.

DDX41-associated familial MDS/AML syndrome: The DDX41-associated familial MDS/AML syndrome is a recently identified autosomal dominant syndrome presenting in mid to late adulthood. This is caused by germline mutations in the DEAD-Box helicase $D D X 41$, leading to altered premRNA splicing and RNA processing [18]. DDX41 mutations result in an increased lifetime risk of myeloid neoplasms, including MDS, AML and CML. The average age at diagnosis is 60 years, making it difficult to clinically distinguish patients with de novo MDS/AML from those with a germline mutation in DDX41. This complication may result in its underdiagnosis. The prevalence of DDX41 germline mutations is not known. However, in a cohort study of 1385 MDS or AML patients, DDX41 variants were identified in 43 patients $(3.1 \%)$, and causal variants were seen in 33 patients $(2.4 \%)$ [19]. These mutations are relatively common in adult MDS/AML, often without a family history, decreasing the need for systemic screening. Other than the significant family history, DDX41-related malignancies have no apparent preceding clinical signs or symptoms related to the increased risk of hematologic malignancy. Similar to other predisposing syndromes, families with known DDX41 mutations require a bone marrow biopsy at diagnosis with cytogenetic analysis and a complete blood count at regular intervals [20].

SRP72-associated MDS: Germline mutations in the ribonucleoprotein complex gene SRP72 have been identified as a rare cause of familial MDS and bone marrow failure. Mutations in SRP72 were detected in two families with aplastic anemia and MDS. In both families, auditory abnormalities were found, and MDS developed in adulthood. Given the rarity of these germline mutations, little is known regarding their incidence and risk for hematologic malignancies [21].

ETV6-associated familial thrombocytopenia and hematologic malignancy: Patients with thrombocytopenia 5 have an autosomal dominant disorder of the thrombocytopenia, leading to increased susceptibility to bleed. Symptoms usually present during childhood, and patients have been found to have germline mutations in ETV6. The common phenotype is mild to moderate thrombocytopenia with a variable predisposition to MDS, AML, and ALL. Recently, Di Paola and Porter [22] reported that thrombocytopenia was almost completely penetrant, and leukemia was reported in around 30\% of carriers with the highest incidence of ALL. Early-onset colorectal cancer has also been reported in a small number of individuals [23].

Detection and management of patients and family members with MDS with genetic predisposition

Any family with a pattern of familial thrombocytopenia and a predisposition to hematological malignancies should be screened for germline mutations. Recognizing individuals with MDS who may harbor germline mutations can be significantly enhanced by obtaining a careful medical and family history. Guidelines for the clinical detection of familial MDS syndromes have been proposed by Churpek et al. [20]. An algorithm for screening newly diagnosed myeloid malignancy patients for further referral to genetic counseling and testing is shown in Fig. 1 [4, 20].

A DNA sample derived from cultured skin fibroblasts remains the gold standard for germline testing. However, if skin fibroblasts are not available, other tissues, such as cultured bone marrow-derived stromal cells, may be used, depending on the laboratory. Also, segregating mutations can be screened if DNA samples are obtained from multiple family members. There is an increasing availability of multigene panel testing for cancer predispositions and platelet disorders. A significant challenge for diagnostic laboratories and clinicians is the classification of variants, which is especially difficult for rare genetic diseases with evolving phenotypes.

Once there is evidence for a pathogenic germline mutation, patients should undergo periodic follow-up with a complete blood count, clinical examination, and a bone marrow biopsy at baseline. A careful clinical examination and regular screening for associated malignancies are recommended, and genetic counseling and site-specific testing should be offered to any family member at risk.

In contrast to sporadic or primary MDS, where the prognostic risk stratification guides the treatment plan, current MDS prognostic models have excluded patients with known

Patients with newly diagnosed MDS

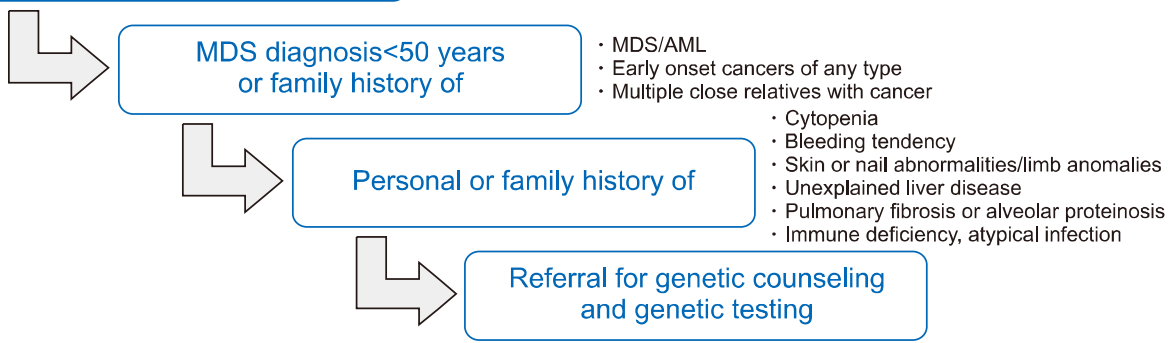

Fig. 1. Algorithm for the genetic evaluation of myelodysplastic syndrome with genetic predisposition. 
genetic predisposition syndromes, greatly underestimating the risk of progression in most cases [24]. The long-term outcomes of MDS secondary to familial MDS/AML predisposition are historically poor, driven by progressive cytopenia, infectious complications, and evolution to acute leukemia. For these patients, HSCT should be considered earlier in the disease course [25, 26]. Any consideration for cytotoxic therapy prior to HSCT must be weighed with the risks of treatment-related toxicity. Further, familial MDS/AML predisposition adds several considerations to the pre-transplant planning and post-HSCT care of an MDS patient, particularly on the choice of a conditioning regimen, selection of HSCT donor, and unique-transplant-related toxicities. Matched-related stem cell donors should be considered very carefully, and donors with known germline mutation or unclear carrier status should be avoided.

\section{CONCLUSIONS}

Increasing awareness about germline predisposition and the widespread application of unbiased whole-exome sequencing has contributed to the discovery of new clinical entities with high risks of developing into hematopoietic malignancies with genetic predispositions. The identification and management of individuals with genetic predisposition is a current challenge for hematologists. Importantly, several features such as familial and personal history and molecular and cytogenetic findings may help clinicians consider an underlying genetic predisposition. It is strongly recommended that precise diagnosis, genetic counseling, familial screening, and follow-up programs be provided for patients predisposed to such malignancies.

\section{Authors' Disclosures of Potential Conflicts of Interest}

No potential conflicts of interest relevant to this article were reported.

\section{REFERENCES}

1. Heaney ML, Golde DW. Myelodysplasia. N Engl J Med 1999; 340:1649-60

2. Swerdlow SH, Campo E, Pileri SA, et al. The 2016 revision of the World Health Organization classification of lymphoid neoplasms. Blood 2016;127:2375-90.

3. University of Chicago Hematopoietic Malignancies Cancer Risk Team. How I diagnose and manage individuals at risk for inherited myeloid malignancies. Blood 2016;128:1800-13.

4. Bannon SA, DiNardo CD. Hereditary predispositions to myelodysplastic syndrome. Int J Mol Sci 2016;17:838.

5. Arber DA, Orazi A, Hasserjian R, et al. The 2016 revision to the World Health Organization classification of myeloid neoplasms and acute leukemia. Blood 2016;127:2391-405.

6. Döhner H, Estey E, Grimwade D, et al. Diagnosis and management of AML in adults: 2017 ELN recommendations from an international expert panel. Blood 2017;129:424-47.

7. Greenberg PL, Stone RM, Al-Kali A, et al. Myelodysplastic syndromes, version 2.2017, NCCN Clinical Practice Guidelines in Oncology. J Natl Compr Canc Netw 2017;15:60-87.

8. Churpek JE, Pyrtel K, Kanchi KL, et al. Genomic analysis of germ line and somatic variants in familial myelodysplasia/acute myeloid leukemia. Blood 2015;126:2484-90.

9. Owen CJ, Toze CL, Koochin A, et al. Five new pedigrees with inherited RUNX1 mutations causing familial platelet disorder with propensity to myeloid malignancy. Blood 2008;112:4639-45.

10. Michaud J, Wu F, Osato M, et al. In vitro analyses of known and novel RUNX1/AML1 mutations in dominant familial platelet disorder with predisposition to acute myelogenous leukemia: implications for mechanisms of pathogenesis. Blood 2002;99: 1364-72.

11. Preudhomme C, Renneville A, Bourdon V, et al. High frequency of RUNX1 biallelic alteration in acute myeloid leukemia secondary to familial platelet disorder. Blood 2009;113:5583-7.

12. Balduini A, Raslova H, Di Buduo CA, et al. Clinic, pathogenic mechanisms and drug testing of two inherited thrombocytopenias, ANKRD26-related thrombocytopenia and MYH9-related diseases. Eur J Med Genet 2018;61:715-22.

13. Noris P, Perrotta S, Seri M, et al. Mutations in ANKRD26 are responsible for a frequent form of inherited thrombocytopenia: analysis of 78 patients from 21 families. Blood 2011;117:6673-80.

14. Noris P, Favier R, Alessi MC, et al. ANKRD26-related thrombocytopenia and myeloid malignancies. Blood 2013;122:1987-9.

15. Sahoo SS, Kozyra EJ, Wlodarski MW. Germline predisposition in myeloid neoplasms: unique genetic and clinical features of GATA2 deficiency and SAMD9/SAMD9L syndromes. Best Pract Res Clin Haematol 2020;33:101197.

16. Spinner MA, Sanchez LA, Hsu AP, et al. GATA2 deficiency: a protean disorder of hematopoiesis, lymphatics, and immunity. Blood 2014;123:809-21.

17. McReynolds LJ, Yang Y, Yuen Wong H, et al. MDS-associated mutations in germline GATA2 mutated patients with hematologic manifestations. Leuk Res 2019;76:70-5.

18. Polprasert C, Schulze I, Sekeres MA, et al. Inherited and somatic defects in DDX41 in myeloid neoplasms. Cancer Cell 2015;27: 658-70.

19. Sébert $M$, Passet $M$, Raimbault $A$, et al. Germline DDX41 mutations define a significant entity within adult MDS/AML patients. Blood 2019;134:1441-4.

20. Churpek JE, Lorenz R, Nedumgottil S, et al. Proposal for the clinical detection and management of patients and their family members with familial myelodysplastic syndrome/acute leukemia predisposition syndromes. Leuk Lymphoma 2013;54:28-35.

21. Kirwan M, Walne AJ, Plagnol V, et al. Exome sequencing identifies autosomal-dominant SRP72 mutations associated with familial aplasia and myelodysplasia. Am J Hum Genet 2012;90: 888-92.

22. Di Paola J, Porter CC. ETV6-related thrombocytopenia and leukemia predisposition. Blood 2019;134:663-7.

23. Zhang MY, Churpek JE, Keel SB, et al. Germline ETV6 mutations in familial thrombocytopenia and hematologic malignancy. Nat Genet 2015;47:180-5. 
24. Bejar R. Prognostic models in myelodysplastic syndromes. Hematology Am Soc Hematol Educ Program 2013;2013:504-10.

25. MacMillan ML, Wagner JE. Haematopoeitic cell transplantation for Fanconi anaemia- when and how? Br J Haematol 2010;149:
14-21.

26. Babushok DV, Bessler M. Genetic predisposition syndromes: when should they be considered in the work-up of MDS? Best Pract Res Clin Haematol 2015;28:55-68. 